\title{
Zur Deutung der Höhenstrahlung ${ }^{1}$
}

\author{
Von Pascual Jordan \\ z. Zt. in Göttingen \\ (Z. Naturforschg. 1, 301-304 [1946]; eingegangen am 31. Oktober 1945)
}

\begin{abstract}
Es wird die Möglichkeit erörtert, die Höhenstrahlung zu deuten als eine ,schwarze“ Strahlung sehr hoher Temperatur ( $\approx 10^{12}$ Grad), emittiert von gewissen sehr heißen Sternen (Supernovastadium). Man hat dann das empirische Spektrum der Höhenstrahlung als Sonderfall eines schwarzen Spektrums anzusehen, und benötigt eine diesen Fall mit umfassende Verallgemeinerung der Pla $\mathrm{nck}$ schen Formel. Eine derartige Verallgemeinerung wird angegeben und ihre Folgerungen werden besprochen: Insbesondere ergibt sich die Existenz einer nicht erreichbaren oberen Grenztemperatur.
\end{abstract}

$I^{\prime \prime}$

m Zusammenhange kosmologisch-astrophysikalischer Überlegungen ${ }^{2}$ habe ich einerseits über die Höhenstrahlung, andererseits über die thermodynamische Rolle der Elementarlänge $l \approx 2 \cdot 10^{-13}$ cm eínige Bemerkungen vorgetragen, die mir heute nicht mehr befriedigend scheinen. Eine veränderte Beurteilung der Sachlage soll im folgenden dargelegt werden.

§1. Das kosmische Ruhesystem. Obwohl uns die spezielle Relativitätstheorie gelehrt hat, daß alle physikalischen Naturgesetze unabhängig von der Wahl des Inertialsystems' sind, so ist doch in jener speziellen Lösung der physikalischen Grundgleichungen, als welche wir den tatsächlichen $\mathrm{Zu}$ stand des Kosmos aufzufassen haben, ein bestimmtes Koordinatensystem $\Sigma_{0}$ gegenüber allen anderen ausgezeichnet, nämlich dasjenige, in welchem sowohl der Schwerpunkt der in Nähe der Milchstraße befindlichen Spiralnebel als auch der Schwerpunkt aller bislang teleskopisch erfaßten Spiralnebel ruht. Man kann $\Sigma_{0}$ auch als dasjenige Koordinatensystem kennzeichnen; in welchem das Strahlungsfeld der Höhenstrahlung isotrop ist.

Für das Ziel unserer Überlegungen ist die Frage wichtig, ob dieses ,kosmische Ruhesystem " $\Sigma_{0}$ auch in physikalischen Elementargesetzen eine Rolle spielen kann; und eine Bejahung wird nur dann

1 Eine vorläufige Mitteilung zu dieser Arbeit erschien in den Göttinger Nachrichten.

2 Physik Z. 45, 183, 233 [1944]. erlaubt sein, wenn eine Entscheidung über den $\mathrm{Zu}$ stand der Ruhe oder Bewegung gegenüber diesem Koordinatensystem $\Sigma_{0}$ grundsätzlich auch ohne Bezugnahme auf das System der Spiralnebel oder die Höhenstrahlung, durch terrestrische Experimente, möglich wäre. Die Diracsche Idee, daß die Gravitationskonstante $*$ in Wahrheit nicht konstant sei, sondern umgekehrt proportional dem Weltalter abnehme, bietet hierfür eine Möglichkeit. Nach dieser Vorstellung ist $x$ eine skalare Feldgröße $x=$ $\varkappa(x, y, z, t)$, welche gerade im Koordinatensystem $\Sigma_{0}$ die Gestalt

$$
\varkappa(x, y, z, t)=\frac{\text { const }}{t}
$$

annimmt, aber in jedem gegen $\Sigma_{0}$ bewegten Koordinatensystem nicht nur zeitlich, sondern auch räumlich inkonstant ist. Der Vierervektor grad $x$ hat also in $\Sigma_{0}$ Komponenten $\left(0,0,0, \frac{\text { Const }}{t^{2}}\right)$; und es gibt einen Vierervektor, der in $\Sigma_{0}$ eine konstante Zeitkomponente hat:

$$
\begin{gathered}
\frac{\operatorname{grad} x}{\varkappa^{2}}=\left(0,0,0, \frac{\dot{x}}{c x^{2}}\right), \\
\frac{\dot{x}}{c x^{2}}=\frac{h}{c l^{3}} z ; z \approx 1 .
\end{gathered}
$$

Wir dürfen es deshalb als erlaubt ansehen, in physikalischen Grundgesetzen der Lichtquantentheorie nicht nur die vom Koordinatensystem abhängige Frequenz $v$ eines Lichtquants auftreten $z u$ lassen, sondern auch denjenigen Wert $\nu^{*}$, welchen $v$ 
im System $\Sigma_{0}$ annimmt: Denn $\nu^{*}$ ist der Wert der relativistischen Invarianten

$$
v^{*}=\frac{l^{3}}{z h x^{2}} \cdot v \cdot\left(\mathfrak{g r a d} x-\frac{1}{c} \frac{\partial \varkappa}{\partial t}\right),
$$

wo der Einheitssektor $\mathfrak{g}$ die Flugrichtung des Lichtquants bezeichnet.

Im folgenden beziehen wir uns stets auf das Koordinatensystem $\Sigma_{0}$, und schreiben dann einfach $\boldsymbol{v}$ statt $\boldsymbol{\nu}^{*}$.

§ 2. Die Eulersche Formel. Die primäre Höhenstrahlung, wie sie von außen in die Atmosphäre eindringt - auf ihre Zusammensetzung aus Protonen oder Elektronen und Lichtquanten usw. gehen wir nicht näher ein - zeigt eine Energiedichte $\varrho_{v} d v$ für das Frequenzintervall $v, v+d v$, welche in einem weiten Bereiche durch die Eulersche Formel

$$
\varrho_{\nu}=\frac{\text { const }}{\nu^{s}} ; s \cong 1,85
$$

beschrieben wird. Diese Formel gilt für Werte $h v$ zwischen $10^{9}$ oder $10^{10}$ bis mindestens $10^{16}$ Elektronvolt.

Wir sind durch diese $\mathrm{F}$ ormel vor ein sehr eigentümliches Problem gestellt, dessen Schwierigkeit übrigens in keiner Weise verringert würde, wenn wir den Entstehungsvorgang der Höhenstrahlung in eine ferne Vorzeit zurückverlegen wollten. Bei genauerer Prüfung scheint es aussichtslos, die Höhenstrahlung auf etwas anderes zurückführen zu wollen, als auf Vorgänge, bei denen sehr hohe Temperaturen aufgetreten sind. Die Gültigkeit der Eulerschen Formel bis $h v \geq 10^{16}$ Elektronvolt macht es aber unmöglich, bei Aufrechterhaltung der gewohnten Boltzmannschen Statistik mit Temperaturen auszukommen, deren $k T$ merklich kleiner als $10^{16}$ Elektronvolt wäre. Aber eine solche Temperatur $T \geq 10^{20}$ Grad würde, wenn sie irgendwann einmal verwirklicht gewesen wäre, ungeheure Beträge auch an langwelligerer Strahlung hinterlassen haben, die tatsächlich keineswegs vorhanden sind.

Es scheint deshalb die Folgerung unausweichlich, daß bei den hohen Temperaturen, welche für die Entstehung der Höhenstrahlung verantwortlich zu machen sind, die bekannten thermodynamisch-statistischen Gesetze versagen, und daß insbesondere eine Abänderung der Planckschen Strahlungsformel

$$
\varrho_{v}=\frac{\operatorname{const} v^{3}}{\exp \left(\frac{h v}{k T}\right)-1}
$$

erforderlich ist. Wir wollen also die Euler sche Formel (4) so deuten, daß sie - analog der Planckschen Formel (5) - das Spektrum eines gewissen Falles schwarzer Strahlung darstellt, und zwar einer Strahlung sehr hoher Temperatur, während sich die Gültigkeit der Planckschen Formel auf wesentlich tiefere Temperaturen (z. B. $T<10^{8}$ Grad) beschränkt. Der Zahlwert $s \simeq 1,85$ legt die Vermutung nahe, daß es sich hier um einen speziellen Zahlwert für eine Veränderliche, also eine Funktion der Temperatur handele.

Es entsteht also die Aufgabe, durch Interpolation eine neue, umfassendere Formel zu bestimmen, welche sowohl die Plancksche als auch die Eulersche als Grenzfälle in sich enthält. Dafür gibt es nun eine bestimmte Möglichkeit, die durch ihre Einfachheit als eindeutige Lösung der gestellten Aufgabe gekennzeichnet scheint:

$$
\varrho_{v}=\frac{\text { const } v^{3}}{\left(1+v / v_{m}\right)^{\Theta} \mp 1} ;
$$

dabei soll $\quad v_{m} l=c ; \frac{h v_{m}}{k T_{m}}=1 ; \Theta=\frac{T_{m}}{T}$

sein. Wir haben \pm 1 geschrieben, um in (6) auch gleich den Fall einer Strahlung von Teilchen mit halbzahligem Spin zu berücksichtigen. Die $R u h$ masse der fraglichen Teilchen soll aber in (6) ausdrücklich $=0$ vorausgesetzt werden; den Fall \pm 0 besprechen wir später gesondert.

§3. Folgerungen. Wir sehen jetzt (6), (7) als gegeben an und betrachten die sich ergebenden Folgerungen.

Für $T \rightarrow \hat{v}$ wird, wie der III. Hauptsatz es verlangt, $\rho_{\nu} \rightarrow 0$. Mit wachsendem $T$ nimmt $\rho_{v}$ (bei festem $v$ ) monoton zu. Bei festem $T$ verschwindet $\varrho_{v}$ für $v=0$, wächst dann bis zu einem Maximum und nimmt danach monoton asymptotisch zu Null ab.

Ist $\nu \ll v_{m}$, so wird bei großem $\bar{\Theta}\left(\right.$ also bei $\left.T \ll T_{m}\right)$ :

$$
\left(1+\frac{v}{v_{m}}\right)^{\Theta} \rightarrow \exp \left(\frac{v}{v_{m}} \Theta\right)=\exp \left(\frac{h v}{k T}\right)
$$

es ergibt sich also für $\rho_{\nu}$ die Planck sche Formel. Is't $v \geqslant v_{m}$, so wird aus (6) die Eu ler sche Formel:

$$
\varrho_{v}=\frac{\text { const }}{v \Theta-3} \text {. }
$$

Hier ergibt sich eine grundsätzliche Folgerung: Damit

$$
u=\int_{0}^{\infty} \varrho_{v} d v
$$


endlich bleibt, muß $\Theta>4$ sein. Somit ergibt sich aus (6) als Folgerung jenes neue thermodynamische Prinzip, welches in früheren Betrachtungen (a.a.O.) vermutungsweise ausgesprochen wurde:

Es gibt eine endliche Höchsttemperatur, die ebenso unerreichbar ist, wie andererseits $T=0$. Ihr Wert ist gegeben durch $\Theta>4$ oder

$$
T<\frac{1}{4} T_{m}
$$

Während aber bei $T \rightarrow \frac{1}{4} T_{m}$ nach (9) die Energiedichte $u$ unendlich wird, bleibt die Gesamtzahl $n$ der im $\mathrm{cm}^{3}$ vorhandenen Teilchen endlich; sie nähert sich einem Grenzwert

$$
n_{m}=\int_{0}^{\infty} \frac{\text { const } \cdot v^{2} d v}{\left(1+\frac{v}{v_{m}}\right)^{4} \mp 1} \approx \text { const } l^{-3} .
$$

Offenbar können also (im Koordinatensystem $\Sigma_{0}$ ) größenordnungsmäßig nicht mehr als 1 Elementarteilchen in einem Elementarvolum $l^{3}$ zusammengedrängt werden. Dieses sehr anschauliche Ergebnis ist auch in Einklang mit der Heisenbergschen Vorstellung ${ }^{3}$, daß die Wirkungsquerschnitte aller Elementarteilchen (im Schwerpunkt-Koordinatensystem des jeweiligen Stoßvorganges') bei großen Energieumsätzen gegen $l^{2}$ konvergieren.

$\S 4$. Entropie. Aus $\rho_{\nu}$ und $v$ kann man die relativistisch invariante Größe $\rho_{\nu} / \nu^{3}$ bilden; Division mit einem geeigneten Zahlfaktor ergibt dann die mittlere Anzahl $n v$ von Strahlungsteilchen für die in Nähe von $v$ liegenden Eigenfrequenzen des Strahlungshohlraums:

$$
n_{v}=\frac{1}{\left(1+\frac{v}{v_{m}}\right)^{\Theta} \mp 1} .
$$

Die zugehörigen Entropie $s_{v}=s\left(n_{v}\right)$ berechnet sich aus der Bedingung, daß

$$
\frac{d s_{v}}{d n_{v}}=\frac{h v}{T}
$$

werden muß. Die Berechnung ist ganz analog der bisherigen Theorie und ergibt

$$
s_{\nu}=\frac{\frac{v}{v_{m}}}{\ln \left(1+\frac{v}{v_{m}}\right)}\left\{ \pm\left(1+n_{v}\right) \ln n_{\nu}-n_{v} \ln n v\right\} ;
$$

für $v \ll v_{m}$ wird der Faktor vor der Klammer gleich 1, und wir kommen zu den gewohnten Formeln zurück.

3 W. Heisenberg, Z. Physik 120, 513 [1943].
Die gesamte räumliche Entropiedichte wird

$$
s=\mathrm{const} \int_{0}^{\infty} v^{2} s_{\nu} d v
$$

für $T \rightarrow \frac{1}{4} T_{m}$ geht $s$ nach $\infty$.

Wir erwähnen noch das mittlere Schwankungsquadrat

$$
\overline{\left(\Delta n_{v}\right)^{2}}=\frac{k T^{2}}{h \nu} \cdot \frac{d n_{v}}{d T},
$$

für das wir aus (13) das Ergebnis

$$
\overline{\left(\Delta n_{v}\right)^{2}}=\frac{\ln \left(1+\frac{v}{v_{m}}\right)}{\frac{v}{v_{m}}} n_{\nu}\left(1 \pm n_{v}\right)
$$

finden, was sich ebenfalls nur durch den vorderen Faktor von der bisherigen Formel $n_{\nu}\left(1 \pm n_{v}\right)$ unterscheidet.

Bislang haben wir die Ruhmassen der betrachteten Teilchen als verschwindend vorausgesetzt. Haben wir es jedoch mit Teilchen endlicher Ruhmasse $m_{0}$ zu tun, so ist einerseits der Faktor $\nu^{2}$ im Integral (16) und in

$$
n=\mathrm{const} \int_{0}^{\infty} v^{2} n_{\nu} d v
$$

in bekannter Weise durch einen allgemeineren zu ersetzen - hierauf braucht nicht näher eingegangen zu werden. Außerdem aber müssen wir dann $v$ in (15) ersetzendurch $v-m_{0} c^{2} / h$. Denn wir müssen ja den von $v$ abhängigen Faktor in $s_{\nu}$ gleich 1 machen, sobald die kinetische Energie $h v-m_{0} c^{2}$ klein gegen $h v_{m}$ wird.

Wir haben also jetzt statt (15) die allgemeinere Formel

$$
s_{\nu}=\frac{\frac{v-m_{0} c^{2} / h}{v_{m}}}{\ln \left(1+\frac{\nu-m_{0} c^{2} / h}{v_{m}}\right)}\left\{ \pm\left(1 \pm n_{\nu}\right) \ln \left(1 \pm n_{\nu}\right)-n_{\nu} \ln n_{\nu}\right\}
$$

und daraus ergibt sich statt (13) die etwas kompliziertere Formel

$$
n_{v}=\frac{1}{\left(1+\frac{v-m_{0} c^{2} / h}{v_{m}}\right) \exp \left(\frac{v \Theta}{v-m_{0} c^{2} / h}\right) \mp 1} .
$$

$\S 5$. Kinetik. Bekanntlich ist das thermodynamische Gleichgewicht in einem Gemisch von quan- 
tenstatistischen Gasen auch an Hand einfacher kinetischer Betrachtungen zu erweisen, welche aus' den ursprünglichen Einsteinschen Überlegungen durch verschiedene Verfasser entwickelt sind ${ }^{4}$.

Bei einem Elementarvorgang

$$
\mathfrak{T}_{1}+\mathfrak{T}_{2}+\ldots \rightarrow \mathfrak{T}_{1}+\mathfrak{T}_{2}{ }_{2}+\ldots
$$

mögen Teilchen $\mathfrak{I}_{1}, \mathfrak{I}_{2}, \ldots$, welche die Energien $h v_{1}, h v_{2}, \ldots$ und die Ruhmassen $m_{1}, m_{2}, \ldots$ besitzen, ursprünglich vorhanden sein; die Teilchen $\mathfrak{I}_{1}{ }^{\prime}$, $\mathfrak{I}_{\mathbf{2}}{ }^{\prime}, \ldots$ seien im Ergebnis des Elementarvorgangs vorhanden. Dann ist die invariante Häufigkeit dieses Elementarvorgangs nach der bisherigen Theorie proportional mit

$$
\vec{W}=n_{1} n_{2} \ldots\left(1 \pm n_{1}^{\prime}\right)\left(1 \pm n_{2}^{\prime}\right) \ldots,
$$

und die geforderte Übereinstimmung mit der entsprechenden Häufigkeit $W$ für den inversen Vorgang verlangt

$$
\left(\frac{1}{n_{1}} \pm 1\right)\left(\frac{1}{n_{2}} \pm 1\right) \cdots=\left(\frac{1}{n_{1}^{\prime}} \pm 1\right)\left(\frac{1}{n_{2}^{\prime}} \pm 1\right) \cdots
$$

oder

$$
\exp \left(\frac{h v_{1}}{k T}\right) \exp \left(\frac{h v_{\circ}}{k T}\right) \ldots=\exp \left(\frac{h v_{1}^{\prime}}{k T}\right) \exp \left(\frac{h v_{2}^{\prime}}{k T}\right) \ldots,
$$

was wegen des Energiesatzes

$$
h v_{1}+h v_{2}+\ldots=h v_{1}^{\prime}+h v_{2}^{\prime}+\ldots
$$

zutreffend ist.

Es ist aber wichtig, zu betonen, daß die Beteiligung eines Teilchens an einem Elementarvorgang nicht notwendigerweise voraussetzt, daß eine tatsächliche Energie-Impulsänderung dieses Teilchens eintritt. Es könnten in (22) auch gewisse Teilchen in stiller (oder „katalytischer") Weise beteiligt sein, derart, daß z. B. $\mathfrak{I}_{1}^{\prime}$ das unbeeinflußte Teilchen $\mathfrak{I}_{1}$ ist. Wir dürfen deshalb (23) erweitern zu

$$
\left.\begin{array}{c}
\vec{W}={ }_{1} n^{1+a_{1}}\left(1 \pm n_{1}\right)^{a_{1}} \cdot n_{2}{ }^{1+a_{2}}\left(1 \pm n_{2}\right)^{a} \ldots \\
\left(1 \pm n_{1}^{\prime}\right)^{1+a_{1}^{\prime}}{ }_{{ }_{1}^{\prime}{ }_{1}^{\prime}}^{a_{1}^{\prime}} \cdot\left(1 \pm n_{2}^{\prime}\right)^{1+a_{2}^{\prime}{ }_{n_{2}^{\prime}}{ }^{\prime}{ }_{2}^{\prime}{ }_{2} \ldots}
\end{array}\right\}
$$

${ }_{4}$ W. Pauli jr., Z. Physik 18, 272 [1923]; A. Einstein u. P. Ehrenfest, Z. Physik 19, 301 [1923]; P. A. M. Dirac, Proc. Roy. Soc. [London] (A) 106, 581 [1924]; J or dan, Z. Physik 33, 649 [1925]; 41, 711 [1927]); W. B othe, Z. Physik 46, 327 [1927].
Nunmehr haben wir (23), (27) so abzuändern, daß statt der bisherigen die neuen Spektralformeln thermodynamisch stationär werden. Dabei gebrauchen wir die Abkürzungen

$$
\left.\begin{array}{c}
v_{j}-m_{j} c^{2} / h=\overline{v_{j}} ; v_{j}^{\prime}-m_{j}^{\prime} c^{2} / h=\overline{v_{j}^{\prime}} ; \\
L(v)=\frac{\frac{v}{v_{m}}}{\ln \left(1+\frac{v}{v_{m}}\right)} .
\end{array}\right\}
$$

Wir verallgemeinern (23) $\mathrm{zu}$

$(\vec{W})^{L}=n_{1}{ }^{L\left(\bar{v}_{1}\right)} n_{2}{ }^{\left(L \bar{v}_{2}\right)} \ldots\left(1 \pm n_{1}^{\prime}\right)^{L\left(\bar{v}_{1}^{\prime}\right)}\left(1 \pm n_{2}^{\prime}\right)^{L\left(\bar{v}_{2}^{\prime}\right)} \ldots$

mit einem noch offen gelassenen Wert $L$. Die entsprechende Verallgemeinerung kann an (27) ausgeführt werden; das Aufschreiben der entstehenden etwas umfangreichen Formel darf unterlassen werden. In jedem Falle gibt die Forderung $\vec{W}=\overleftarrow{W}$ bei einer Verteilung (21) wieder die erfüllte Gleichung (25), (26).

Empirisch prüfbar sind nur solche Fälle, bei denen ein einzelnes energiereiches Teilchen (Energie $h \nu_{1}$ ) mit einem langsamen Teilchen etwa der Luft oder in einer Pb-Platte (Energie $h \nu_{2}$ ) zusammenstößt, wonach irgendwelche Teilchen $h \boldsymbol{\nu}_{1}{ }^{\prime}$, $h v_{2}{ }^{\prime}, \ldots$ auftreten. Die Wahrscheinlichkeit dafür ist proportional $n_{1}, n_{2}, \ldots$, und wir haben uns zu überzeugen, daß das im Rahmen unserer Ansätze möglich ist. In der Tat erhalten wir das Gewünschte durch den Ansatz

$$
\left.\begin{array}{c}
(\vec{W})^{L}=n_{1} n_{2}\left(1 \pm n_{1}\right)^{L-L\left(\overline{v_{1}}\right)}\left(1 \pm n_{2}\right)^{L-L\left(\overline{v_{2}}\right)} \cdot \\
\cdot\left(1 \pm n_{1}^{\prime}\right)^{L\left(\bar{v}_{1}^{\prime}\right)}\left(1 \pm n_{2}^{\prime}\right)^{L\left(\bar{v}_{2}^{\prime}\right)} \cdots
\end{array}\right\}
$$

Diese Betrachtungen werden zwar erst dann voll befriedigen können, wenn ihre deduktive Herleitung aus allgemeinen Prinzipien gelungen sein wird: Immerhin zeigen sie bereits; daß der hier in Erwägung gezogene Gedanke mit den heute vorhandenen Erfahrungstatsachen lückenlos harmoniert. 